\title{
The continuity of prime numbers can lead to even continuity(The ultimate)
}

\section{Xie Ling ( 29997609@qq.com )}

Cosmic Mathematical Physics

\section{Research Article}

Keywords: prime even continuity, Bertrand Chebyshev theorem, Ascending and descending, Extreme law

Posted Date: July 27th, 2021

DOI: https://doi.org/10.21203/rs.3.rs-713902/v5

License: (a) (i) This work is licensed under a Creative Commons Attribution 4.0 International License. Read Full License 


\section{The continuity of prime numbers can lead to even continuity(The ultimate)}

Author: Xie Ling

Absrtact : $n$ continuous prime numbers can combine a group of continuous even numbers. If an adjacent prime number is followed, the even number will continue.

For example, if we take prime number $\mathbf{3}$, we can get even number $\mathbf{6}$. If we follow an adjacent prime number 5, we can get even numbers by using 3 and 5: 6, 8 and 10.

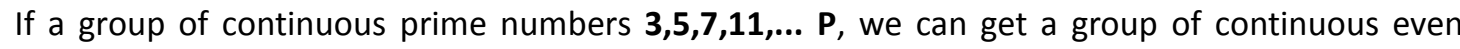
numbers $\mathbf{6 , 8 , 1 0 , 1 2 , \ldots , 2} \mathbf{2}$. Then if an adjacent prime number $q$ is followed, the original group of even numbers $\mathbf{6 , 8}, \mathbf{1 0}, \mathbf{1 2}, \ldots, \mathbf{2 n}$ will be finitely extended to $\mathbf{2}(\mathbf{n}+\mathbf{1})$ or more adjacent even numbers. My purpose is to prove that the continuity of prime numbers will lead to even continuity as long as $\mathbf{2}(\mathbf{n}+\mathbf{1})$ can be extended.

If the continuity of even numbers is discontinuous, it violates the Bertrand Chebyshev theorem of prime numbers.

Because there are infinitely many prime numbers: $\mathbf{3}, \mathbf{5}, \mathbf{7}, \mathbf{1 1}, \ldots$

We can get infinitely many continuous even numbers: $6,8,10,12, \ldots$

Key words: prime even continuity; Bertrand Chebyshev theorem; Ascending and descending; Extreme law; Mathematical complete induction

\section{1}

\section{Preface}

The whole proof begins with the ascent

Rule 1: the minimum odd prime number is 3

Rule 2: add two odd prime numbers.(any combination of two odd primes).

Rule 3: odd prime number can be quoted repeatedly.

Rule 4: meet the previous provisions, and all prime combination to the maximum.

Rule 5: $\mathrm{P}_{\mathrm{a}}+\mathrm{P}_{\mathrm{b}}=2 \mathrm{~S}$, and $\mathrm{P}_{\mathrm{b}}+\mathrm{P}_{\mathrm{a}}=2 \mathrm{~S}$, Delete one and leave only one.

I can quote the minimum odd prime number 3

The results are as follows

$3+3=6$

$\mathbf{5}+\mathbf{1}=\mathbf{6}$ (it is stipulated that $\mathbf{1}$ is not a prime number, which is deleted because it violates regulation 2 )

$\therefore$ The unique formula: $3+3=6$.(comply with Rule 4 : all prime numbers are quoted to the maximum).

According to the rules, the prime number 3 uses the maximum combination, and can only get:

$3+3=6$

How to make 6 continuous to 8 ? 
If we take the adjacent prime number $\mathbf{P}$ greater than 3 , we can get $\mathbf{P}-\mathbf{3} \geqslant \mathbf{2}$

(a)

From $3+3=6, \rightarrow 3+(3+2)=6+2$

If $(\mathbf{3}+\mathbf{2})=\mathbf{P}$, the proposition holds when the even number is $\mathbf{8}$

Extreme law $\rightarrow$ Assumption: $(\mathbf{3}+\mathbf{2}) \neq \mathbf{p}$

$\because \quad p-3 \neq 2$.

$\therefore \quad(a): \quad\{p-3 \geqslant 2, p-3 \neq 2\} \rightarrow(p-3>2) \rightarrow(p-3 \geqslant 4)$

$\therefore p>2 \times 3$

$\therefore p>2 \times 3>3$

The results of Bertrand Chebyshev theorem are as follows:

$(2 \times 3)>\left(\right.$ prime number $\left.\mathbf{p}_{1}\right)>3$.

$\therefore(\mathbf{p}>\mathbf{2} \times \mathbf{3}>\mathbf{3}) \rightarrow(\mathbf{p}>\mathbf{p} \mathbf{1}>\mathbf{3}) \rightarrow(\mathrm{P}$ and 3 are adjacent prime numbers $)$ contradiction. Negation hypothesis.

$\therefore(3+2)=p, \quad \therefore 3+p=8$.

$\therefore \quad 6 \rightarrow 8$

The proof process uses the known prime number, and the sieve method is not used in the derivation process,

Prime number theorem and Bertrand Chebyshev theorem.

By quoting the prime number theorem, we get a finite number of continuous prime numbers, and then generate even numbers according to the requirements of this manuscript.

Use the extreme rule again $\psi$, Force even numbers to continue.

2

nouns and definitions, citing theorems

$\phi_{1} \quad$ Definition of prime number: prime number refers to the natural number with no other factors except 1 and itself in the natural number greater than $\mathbf{1 .}$

$\phi_{2} \quad$ Extreme law : A may or may not be true. What conclusion can we get if we only prove that $\mathbf{A}$ is not true.

$\{\mathbf{A} \mid \mathbf{A}=\mathbf{x}, \mathbf{A}=\mathbf{y}\},(\mathbf{A}=\mathbf{x}) \rightarrow(Q E D)$. Take: $\mathbf{A} \neq \mathbf{x}$, only prove the $\mathbf{A}=\mathbf{y}$ conclusion.

$\phi_{3}$ [References cited 1] Bertrand Chebyshev theorem: if the integer $\mathbf{n}>\mathbf{3}$, then there is at least one prime $\mathbf{P}$, which conforms to $\mathbf{n}<\mathbf{p}<\mathbf{2} \mathbf{n}-\mathbf{2}$. Another slightly weaker argument is: for all integers $n$ greater than $\mathbf{1}$, there is at least one prime $P$, which conforms to $\mathbf{n}<\mathbf{p}<\mathbf{2 n}$.

$\phi_{4} \quad$ In this paper, the even number generation rules are as follows

(1) Only odd primes are allowed as elements.

(2) Only two prime numbers can be added.(any combination of two odd primes).

(3) Two prime numbers can be used repeatedly: $(3+3)$, or $(3+5)$, or $(\mathbf{P}+\mathbf{P})$.

(4) meet the previous provisions, and all prime combination to the maximum.

(5) Take only one of $\left(\mathbf{p}_{\mathbf{a}}+\mathbf{p}_{\mathrm{b}}\right)$ and $\left(\mathbf{p}_{\mathrm{b}}+\mathbf{p}_{\mathbf{a}}\right)$.

$\phi_{5}$ [References cited 2] The theorem of infinite number of primes: 
the $\mathrm{n}$ bit after each prime can always find another prime.

For example, $\mathbf{3}$ is followed by $\mathbf{5}$ and $\mathbf{1 3}$ is followed by $\mathbf{1 7}$; There must be an adjacent prime $\mathbf{p}_{\mathrm{i}}$ after the prime $\mathbf{P}$.

$\phi_{6} \quad$ Here we only discuss the following cases: prime number sequence and even number sequence

$\left(\phi_{5}\right) \rightarrow$ Prime number sequence: $3,5,7,11,13,17,19,23, \ldots$

Even number sequence: $6, \mathbf{8}, \mathbf{1 0}, \mathbf{1 2}, \mathbf{1 4}, \mathbf{1 6}, \mathbf{1 8}, \mathbf{2 0}, \ldots$

Explain $\phi_{6}$ in today's words: the prime number in the prime number sequence discussed in this paper is adjacent and continuous, and the first number is $\mathbf{3}$.

An even number in an even number sequence is contiguous and the first number is 6 .

$\phi_{7} \quad$ Remember: all the primes I'll talk about below refer to odd primes (excluding 2).

Prime symbol $\mathbf{P}$, different primes use $\mathbf{P}_{1}, \mathbf{P}_{2}, \mathbf{P}_{3}, \ldots, \mathbf{p}_{\mathbf{x}}$

3

Logical argument

$\Theta_{1} \quad$ Goldbach conjecture : $3 \leqslant \forall n \in N, \quad 2 n=p_{x}+p_{y}$. set up $\Delta_{1}: p_{x} \geqslant p_{y}$

$\Theta_{2}$ Theorem: the continuity of prime numbers leads to the continuity of even numbers

In mathematical language :

Known: $\left\{\mathbf{3}, \mathbf{5}, \mathbf{7}, \mathbf{1 1}, \mathbf{1 3}, \ldots, \mathbf{p}_{\mathbf{2}}, \mathbf{p}_{\mathbf{1}}, \mathbf{p}_{\mathbf{0}}\right\} \in$ (prime), The next neighbor of $\mathbf{p}_{\mathbf{1}}$ is $\mathbf{p}_{\mathbf{0}}$,

$3<5<7<11<13<\ldots p_{2}<p_{1}<p_{0}$,

$\{6,8,10,12,14,16, \ldots . ., 2 n\} \in$ (continuous even number).

If $:\left\{3,5,7,11,13, \ldots, p_{2}, p_{1}\right\} \rightarrow\{6,8,10,12,14,16, \ldots . ., 2 n\}$.

inevitable: $\left\{3,5,7,11,13, \ldots, p_{2}, p_{1}, p_{0}\right\} \rightarrow\{6,8,10,12,14,16, \ldots . .2 n, 2(n+1)\}$.

prove:

Humans use computers to calculate a finite number of even numbers: $\{6,8,10,12,14, \ldots, 2 n\}$

every even number satisfies $\Theta_{1}$.

The computer process is finite $\{6,8,10,12,14, \ldots, 2 n\}$

It is not logically proved that any even number greater than $\mathbf{4}$ satisfies $\Theta_{1}$.

$\left(\phi_{5}+\phi_{6}\right)$ Take odd prime sequence: $3,5,7,11,13,17,19,23, \ldots$

Take the minimum prime number 3 from the front of the prime sequence,

$\mathbf{A}_{1} \quad\left\{\phi_{1}+\phi_{4}+\{3\}\right\} \rightarrow:$ 
$\{3+3=6\} \quad$ It is recorded as $\mathbf{A}_{1}$

$\rightarrow 6$

According to the rule, 3 can only get $\{3+3=6\}$

Nonexistence: $5+p=6$

I Because $\mathbf{1}$ in $\mathbf{5 + 1 = 6}$ is not defined as a prime number. If $\mathbf{1}$ is defined as a prime number, this paper will come to the same conclusion】

Note: $5 \notin\{3\}$.

Prime number 3 , limit is used according to $\phi_{4}$, cannot be: $6 \rightarrow 8$.

$\left\{\phi_{1}+\phi_{4}+\{3\}\right\} \rightarrow \quad$ : Quoting prime number $\mathbf{3}$ can only get even number $\boldsymbol{6}$

If you want to: $6 \rightarrow 8$, you must add an adjacent prime number 5 .

$\mathbf{A}_{2} \quad\left\{\phi_{1}+\phi_{4}+\{3,5\}\right\} \rightarrow:$

$3+3=6$

$3+5=8$

$5+5=3+7=10 \quad \because \phi_{4}\{(5,5),(3,7)\} \in 10 . \therefore\left\{\phi_{1}+\phi_{4}+\{3,5,7\}\right\} \rightarrow$

$7+5=12$

$7+7=11+3=14 . \because \phi_{4} \quad\{(7,7),(3,11)\} \in 14 . \therefore\left\{\phi_{1}+\phi_{4}+\{3,5,7,11\}\right\} \rightarrow$

$11+5=13+3=16 . \because \phi_{4}\{(11,5),(13,3)\} \in 16 . \therefore\left\{\phi_{1}+\phi_{4}+\{3,5,7,11,13\}\right\} \rightarrow$

$11+7=13+5=18$.

$13+7=17+3=20$. There is a new prime number 17 in continuity.

Note: $\{(\mathbf{1 3}, \mathbf{7}),(17, \mathbf{3})\} \in \mathbf{2 0}$. even numbers $\rightarrow: 6,8,10,12,14,16,18,20$.

Prime numbers are continuous, and there is a new prime number 17.

even numbers $\rightarrow: 6,8,10,12,14,16,18,20$.

Note the key point: $\mathbf{A}_{\mathbf{1}}$ broken, increase the adjacent prime number $\mathbf{5}$ to have $\mathbf{A}_{\mathbf{2}}$

From $\mathbf{A}_{1} \rightarrow \mathbf{A}_{2} \rightarrow$ is it always infinite? Or will it stop?

Here's the wonderful thing:

(analysis I) :

Always Unlimited: $\quad \mathbf{A}_{1} \rightarrow \mathbf{A}_{2} \rightarrow \quad \ldots \ldots$

There are: $\left(\phi_{5}+\phi_{6}\right) \Rightarrow\{3,5,7,11,13,17,19,23, \ldots$

Get: $6,8,10,12,14,16,20,22, \ldots$

Conclusion: $\Theta_{2} \quad$ (QED).

$\left(\phi_{2}\right) \rightarrow$ Stop at $\mathbf{A}_{\mathbf{n}}$, cannot continue. 
(analysis II): Stop at $\mathbf{A}_{\mathbf{n}}$, not to be continued.

Stop at $\quad \mathbf{A}_{\mathbf{n}}: \mathbf{A}_{1} \rightarrow \mathbf{A}_{2} \rightarrow \ldots \ldots . . \mathbf{A}_{\mathbf{n}}$

There are: $3,5,7,11,13,17,19,23, \ldots, p_{2}, p_{1}$

Get: $6,8,10,12,14,16,20,22, \ldots . ., 2(n-1), 2 n$

$\Theta_{3}:\left\{\phi_{1}+\phi_{4}+\left\{3,5,7,11, \ldots, p_{2}, p_{1}\right\}=\triangleright\{6,8,10,12, \ldots . ., 2(n-1), 2 n\}\right.$

$\Theta_{4}:\left\{\phi_{1}+\phi_{4}+\left\{3,5,7,11, \ldots, p_{2}, p_{1}\right\} \neq \triangleright 2(n+1)\right.$

(1): $\quad\left\{\right.$ Let: prime $p \quad$ satisfy: $\left.p \notin\left\{3,5,7,11, \ldots, p_{2}, p_{1}\right\}, p \in \forall \quad \Theta_{4}: p \notin \mathbf{A}_{n}, p_{1}<p.\right\}$

Take the prime number that is greater than $p_{1}$ and adjacent to $p_{1}$ as $p_{0}$,

$\because$ (1) $\rightarrow\left\{p_{0}+3 \neq 2 n, p_{0}+3 \nless 2 n\right\}$

$\therefore \quad p_{0}+3>2 n$

$\therefore p_{0}+2 \geqslant 2 n \quad \because \quad$ (odd) $\neq$ (even)

$\therefore p_{0}+2>2 n \quad \rightarrow p_{0}+1 \geqslant 2 n$

(W)

\section{Starting from (Analysis II)}

The principle of mathematical complete induction: it is correct in the front, until

$\mathbf{A}_{\mathrm{n}}$.

Take the continuous prime number $\left(3,5,7,11, \ldots, \mathbf{p}_{2}, \mathbf{p}_{1}\right)$ from small to large.

$\mathbf{A}_{\mathbf{n}}$

$$
\left\{\phi_{1}+\phi_{4}+\left\{3,5,7,11, \ldots, p_{2}, p_{1}\right\}\right\} \rightarrow \quad:
$$

$\{3+3=6$

$5+3=8$

$7+3=5+5=10$. set up: $(7+3=5+5)$ sequence: $7>5$

$7+5=12$

$11+3=7+7=14$. set up $\Delta_{2}:(11+3=7+7) \quad$ sequence: $11>7$

$13+3=11+5=16$. set up $\Delta_{2}:(13+3=11+5)$ sequence: $13>11$

$13+5=11+7=18 . \quad$ set up $\Delta_{2}:(13+5=11+7)$ sequence: $13>11$

$17+3=13+7=20$. set up $\Delta_{2}:(17+3=13+7)$ sequence: $17>13$

$19+3=17+5=11+11=22$. set up $\Delta_{2}:(19+3=17+5=11+11)$ sequence: $19>17>11$

$19+5=17+7=13+11=24$. set up $\Delta_{2}:(19+5=17+7=13+11)$ sequence: $19>17>13$ 
$p_{e}+p_{f}=2(n-2)$

$p_{c}+p_{d}=2(n-1)$

$\left.p_{1}+p_{b}=2 n\right\} \quad$ It is recorded as $\quad A_{n}$

$\rightarrow: 6,8,10,12,14,16,18, \ldots, 2(n-1), 2 n$.

See $\mathbf{A}_{\mathrm{n}}$ prime numbers of the same color: $3 \rightarrow 5 \rightarrow 7 \rightarrow 7 \rightarrow 11 \rightarrow 13 \rightarrow 13 \rightarrow 17 \rightarrow 19 \rightarrow 19 \ldots . . . \mathrm{p}_{1}$

$\alpha$ line: $3 \rightarrow 5 \rightarrow 7 \rightarrow 7 \rightarrow 11 \rightarrow 13 \rightarrow 13 \rightarrow 17 \rightarrow 19 \rightarrow 19 \rightarrow \ldots . . p_{1}$

Record it as continuous rise: $3 \rightarrow 5 \rightarrow 7$ and $13 \rightarrow 17 \rightarrow 19$. Mold record: $P \rightarrow P+2 a$

Record as continuous platform: $7 \rightarrow 7$. And: $13 \rightarrow 13$, Mold record: $P \rightarrow P$.

Confirm: $(\rightarrow)$ superior subordinate relationship, (lower) $\rightarrow$ (upper).

From what we know now $(a)$ The line is: (lower) $\leqslant$ (upper).

See: $3 \rightarrow 5$ is:(lower) $<$ (upper). $7 \rightarrow 7 \quad$ is: (lower) $=$ (upper).

What man knows: in $\mathbf{A}_{\mathbf{n}}$ a When we reach the last $\mathrm{p}_{1}$, all of them conform to:

(lower) $\leqslant$ (upper)

$\alpha$ Route (limited) ascending and descending in turn:

$\because \quad \mathbf{A}_{1} \rightarrow \mathbf{A}_{\mathbf{n}}$ is a finite number: $3 \rightarrow 5 \rightarrow 7 \rightarrow 7 \rightarrow 11 \rightarrow 13 \rightarrow 13 \rightarrow 17 \rightarrow 19 \rightarrow 19 \ldots . . \mathrm{p}_{1}$

$\therefore \mathbf{A}_{1} \leftarrow \mathbf{A}_{\mathbf{n}}$ is a finite number: $3 \leftarrow 5 \leftarrow 7 \leftarrow 7 \leftarrow 11 \leftarrow 13 \leftarrow 13 \leftarrow 17 \leftarrow 19 \leftarrow 19 \ldots \leftarrow p_{1}$

Regulation (flat form): $\mathbf{p}_{\mathbf{x}} \rightarrow \mathbf{p}_{\mathbf{x}}$

$\mathbf{A}_{\mathbf{n}}$ is simplified as $\mathbf{B}_{\mathbf{n}}$.

$\{3+3=6$

$5+3=8$

$7+3=10$

$7+5=12$

$11+3=14$

$13+3=16$

$13+5=18$

$17+3=20$

$19+3=22$

$19+5=24$

$23+3=26$

$p_{e}+p_{f}=2(n-2)$

$p_{c}+p_{d}=2(n-1)$

$\left.\mathrm{p}_{\mathrm{a}}+\mathbf{p}_{\mathrm{b}}=\mathbf{2 n}\right\} \quad$ It is recorded as $\quad \mathbf{B}_{\mathbf{n}}$ 
Add the number to $\mathbf{B}_{\mathbf{n}}$ and change to: $\mathbf{C}_{\mathbf{n}}$

$\{3+2(n-2)+3=2(n+1)$

$5+2(n-3)+3=2(n+1)$

$7+2(n+4)+3=2(n+1)$

$7+2(n+5)+5=2(n+1)$

$11+2(n-6)+3=2(n+1)$

$p_{e}+6+p_{f}=2(n+1)$

$p_{c}+4+p_{d}=2(n+1)$

$\left.p_{1}+2+p_{b}=2(n+1)\right\} \quad$ It is recorded as $C_{n}$

$\beta$ line: $2 \rightarrow 4 \rightarrow 6 \rightarrow 8 \rightarrow \ldots \rightarrow 2(n-6) \rightarrow 2(n-5) \rightarrow 2(n-4) \rightarrow 2(n-3) \rightarrow 2(n-2)$

$\alpha$ line: $3 \rightarrow 5 \rightarrow 7 \rightarrow 7 \rightarrow 11 \rightarrow 13 \rightarrow 13 \rightarrow 17 \rightarrow 19 \rightarrow 19 \rightarrow \ldots . . p_{1}$

$\boldsymbol{\alpha}$ line: Any two adjacent prime numbers satisfy: (lower) $\leqslant$ (upper).

$\boldsymbol{\alpha}$ line is simplified to $\mathrm{k}$ line: $3 \rightarrow 5 \rightarrow 7 \rightarrow 11 \rightarrow 13 \rightarrow 17 \rightarrow 19 \rightarrow \ldots \rightarrow p_{2} \rightarrow p_{1}$

$\boldsymbol{\alpha}$ line has (T station), (T station): $7 \rightarrow 7,13 \rightarrow 13, \mathbf{p} \rightarrow \mathbf{p}$.

According to ( $k$ line), we get:

$\left\{p_{1}-p_{2}=2 a_{1}\right.$

$P_{2}-p_{3}=2 a_{2}$

$P_{3}-p_{4}=2 a_{3}$

$7-5=2 a_{n}$

$\left.5-3=2 a_{(n+1)}\right\}$ is recorded as: $D_{n}$

\section{Theorem $\left(\omega_{1}\right)$ :}

Known:

$\left\{A_{n}, B_{n}, C_{n}, D_{n}, \phi_{2}, \quad\right.$ The first prime after $p_{1}$ is $p_{0}, p_{0}>p_{1}$,

$\beta$ line: $2 \rightarrow 4 \rightarrow 6 \rightarrow 8 \rightarrow \ldots \rightarrow 2(n-6) \rightarrow 2(n-5) \rightarrow 2(n-4) \rightarrow 2(n-3) \rightarrow 2(n-2)$

$\alpha \quad$ line: $3 \rightarrow 5 \rightarrow 7 \rightarrow 7 \rightarrow 11 \rightarrow 13 \rightarrow 13 \rightarrow 17 \rightarrow 19 \rightarrow 19 \rightarrow \ldots . . p_{1}$

к line: $\left.3 \rightarrow 5 \rightarrow 7 \rightarrow 11 \rightarrow 13 \rightarrow 17 \rightarrow 19 \rightarrow \ldots \rightarrow p_{2} \rightarrow p_{1}\right\}$

There must be: $p_{0}-p_{1}>2(n-2)$

\section{Proof:}

$\because \alpha \quad$ line: Any two adjacent prime numbers satisfy: (lower) $\leqslant$ (upper).

$\because \quad p_{0}>p_{1}$ 


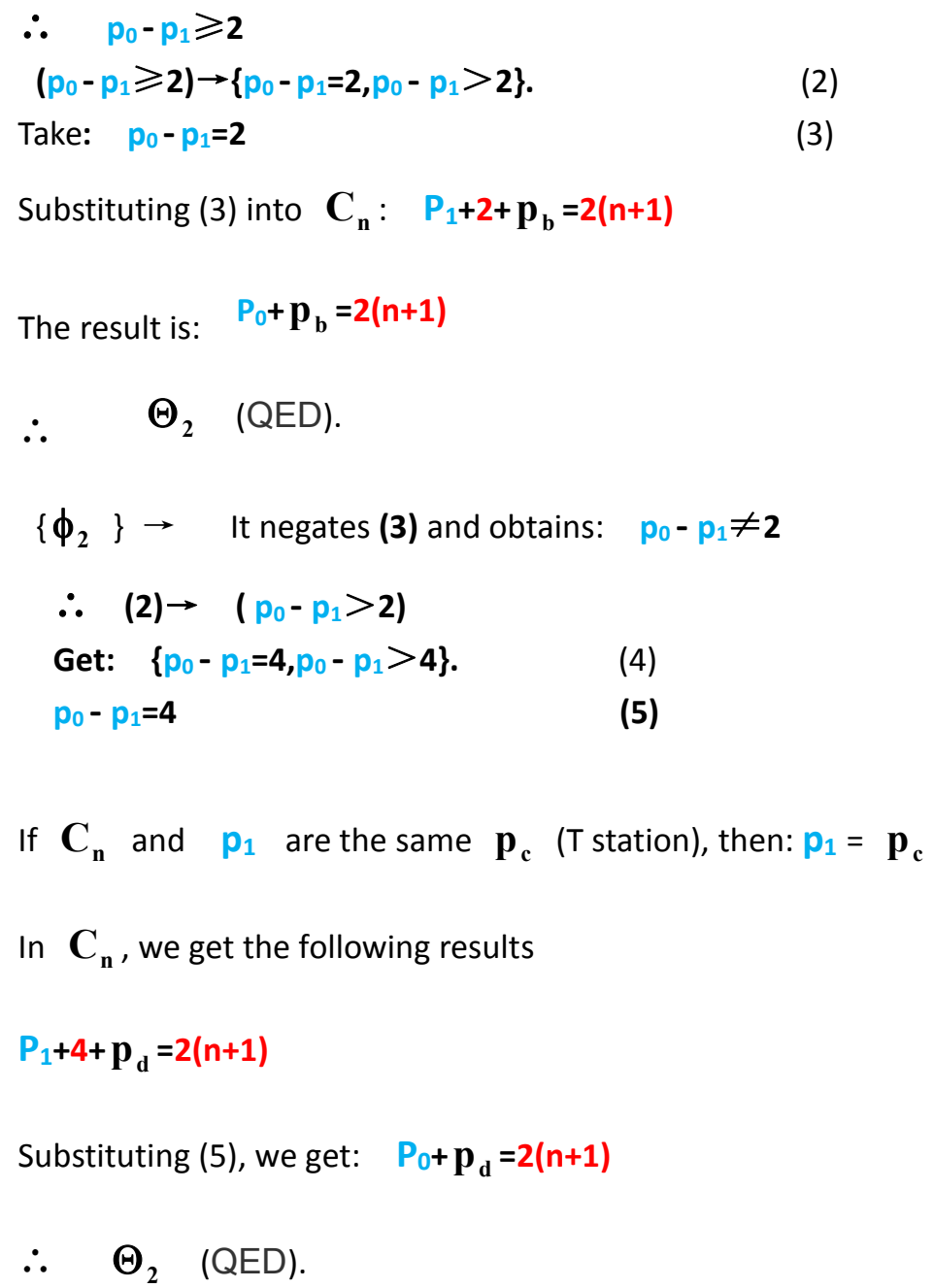

Take: $p_{0}-p_{1}=\mathbf{2}$

Substituting (3) into $\mathbf{C}_{\mathbf{n}}: \mathbf{P}_{1}+2+\mathbf{p}_{\mathbf{b}}=2(\mathbf{n + 1})$

The result is: $\quad P_{0}+\mathbf{p}_{b}=2(n+1)$

$\therefore \quad \Theta_{2}$ (QED).

$\left\{\phi_{2}\right\} \rightarrow \quad$ It negates (3) and obtains: $\quad p_{0}-p_{1} \neq \mathbf{2}$

$\therefore \quad(2) \rightarrow\left(p_{0}-p_{1}>2\right)$

Get: $\left\{p_{0}-p_{1}=4, p_{0}-p_{1}>4\right\}$.

$p_{0}-p_{1}=4$

If $\mathbf{C}_{\mathbf{n}}$ and $p_{1}$ are the same $\mathbf{p}_{\mathbf{c}}$ (T station), then: $p_{1}=\mathbf{p}_{\mathbf{c}}$

In $\mathbf{C}_{\mathbf{n}}$, we get the following results

$P_{1}+4+p_{d}=2(n+1)$

Substituting (5), we get: $\quad \mathbf{P}_{0}+\mathbf{p}_{\mathbf{d}}=\mathbf{2}(\mathrm{n}+1)$

$\therefore \Theta_{2}$ (QED).

$\left\{\phi_{2}\right\}$ negates (5), The result is: $p_{0}-p_{1} \neq \mathbf{4}$.

With the same logic, all the results of the same (T station) are obtained: $p_{0}-p_{1}>\mathbf{2 a} \mathbf{1}$

If $\mathrm{p}_{\mathrm{c}}$ and $\mathrm{p}_{1}$ in $\mathbf{C}_{\mathbf{n}}$ are not in the same (T station).

In (6), a1=1.. Go straight to the next (T station). I'm not sure how big a1 is. The natural number a1 is to be determined.

(T station)in $\mathbf{C}_{\mathbf{n}}$ and $\mathbf{A}_{\mathbf{n}}$,

If: $p_{1}-p_{2}=2 a 1 . \quad$ No (T station): $p_{1}-p_{2}=\mathbf{2}$.

A schematic diagram:

$p_{2}+p_{d}=2(n-1)$

$\left.p_{1}+p_{b}=2 n\right\}$ It is recorded as $A_{n}$

If $p_{1}-p_{2}=2 a 1$, there is (t station) $a 1=2, \quad p_{1}-p_{2}=4$. 
A schematic diagram:

$p_{1}+p_{d}=2(n-1)$

$\left.p_{1}+p_{b}=2 n\right\}$ It is recorded as $A_{n}$

If $p_{1-} p_{2}=2 a 1$, there is (t station) $a 1=3, p_{1}-p_{2}=6$.

$\mathbf{A}_{\text {n }}$ schematic diagram:

$p_{1}+p_{f}=2(n-2)$

$p_{1}+p_{d}=2(n-1)$

$\left.p_{1}+p_{b}=2 n\right\}$ It is recorded as $A_{n}$

If: $p_{1}-p_{2}=2 a 1 . p_{2}-p_{3}=2 a 2 . \quad$ No (T station): $p_{1}-p_{2}=2, p_{2}-p_{3}=2$.

$\mathbf{A}_{\text {n }}$ schematic diagram:

$p_{3}+p_{f}=2(n-2)$

$p_{2}+p_{d}=2(n-1)$

$\left.\mathrm{p}_{1}+\mathbf{p}_{\mathrm{b}}=\mathbf{2 n}\right\}$ It is recorded as $\boldsymbol{A}_{\mathrm{n}}$

The explanation of the schematic diagram is over.
$\ln \mathbf{C}_{\mathbf{n}}: \mathrm{p}_{2}+\mathbf{p}_{\mathrm{s}}=2 \mathrm{n}-2 \mathrm{a}_{1}-2$

$\therefore$ Adjacent to (7): $p_{1}+p_{v}=2 n-2 a_{1}$

From (6): $\quad p_{0}-p_{1}=2 a_{1}+2$

In $\mathbf{D}_{\mathbf{n}}: \quad \mathrm{p}_{1}-\mathrm{p}_{2}=2 \mathrm{a}_{1}$

(9) $\rightarrow\left\{P_{0}-\left(2 n-2 a 1-p_{v}\right)=2 a 1+2\right\}$

The results show that: $P_{0}+\mathbf{p}_{v}=2 n+2 \quad \therefore \quad \Theta_{2} \quad$ (QED).

$\left\{\phi_{2}\right\} \rightarrow$ It negates (9), The results showed that $p_{0}-p_{1}>2 a_{1}+2$

From (6): $p_{0}-p_{1}=2 a_{1}+4$

Similarly, it negates the (11) formula,

The results showed that $p_{0}-p_{1}>2 a_{1}+4$

Similarly, the negative (11) form,

The results are as follows: $p_{0}-p_{1}>2 a_{1}+2 a_{2}+2$

$\because D_{n}+(\beta$ line: 2 (n-2) $)$ 


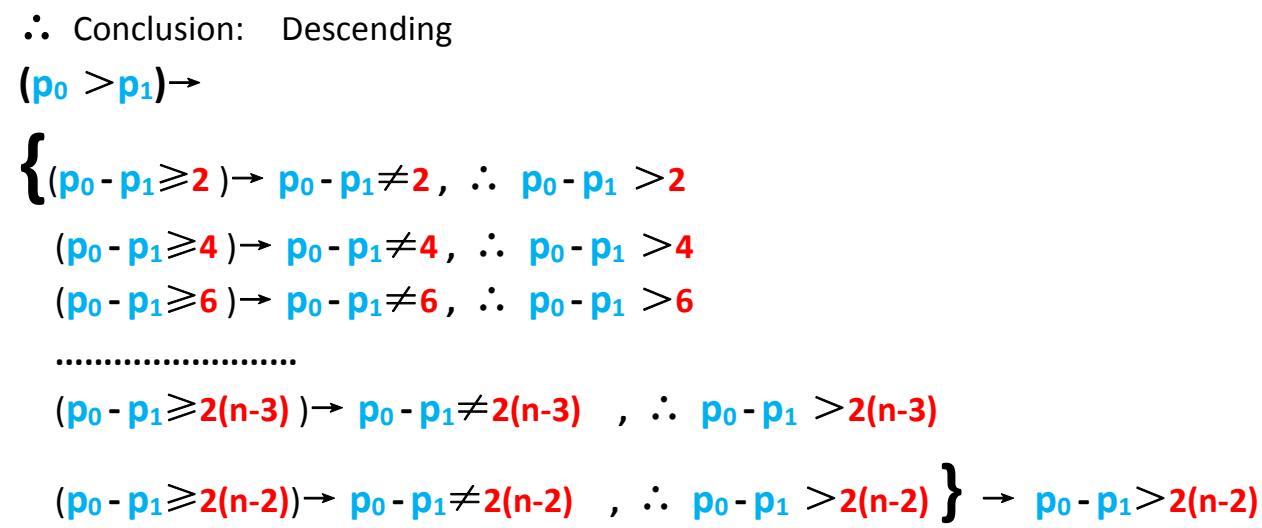

By analogy, we get: $\quad p_{0}-p_{1}>2(n-2) \quad$ Note: $\quad C_{n} \rightarrow \beta$ line: $2(n-2)$

$\left(\omega_{1}\right)$ (QED)

$\left(\omega_{1}\right) \rightarrow\left(p_{0}-p_{1}>2(n-2)\right) \rightarrow\left(p_{0}-p_{1}>2 n-4=p_{1}+p_{b}-4\right)$

$\therefore \quad p_{0}>\left(2 p_{1}+p_{b}-4\right)$

$\therefore \quad p_{0} \geqslant\left(2 P_{1}+p_{b}-3\right)$

$\because$ (the smallest prime in $\mathrm{k}$ is 3$) \rightarrow \mathbf{p}_{\mathrm{b}} \geqslant 3 \quad \therefore\left(p_{0} \geqslant 2 p_{1}+\mathbf{p}_{\mathrm{b}}-3\right) \rightarrow p_{0} \geqslant 2 p_{1}$

$\because$ (odd) $\neq$ (even). $\therefore p_{0}>2 p_{1}$

It is proved that: $p_{0}>2 P_{1}$

$\therefore \quad p_{0}>2 P_{1}>P_{1}$

$\phi_{3} \rightarrow p_{0}>2 p_{1}>p_{g}>p_{1}$

Get: $\quad\left(p_{0}>\mathbf{p}_{g}>p_{1}\right) \rightarrow$ And $p_{0}, p_{1}$ are adjacent, contradictory.

It is proved that it is wrong to quote "Extreme law $\phi_{2}$ " in the process.

Get: $\mathrm{p}_{\mathrm{x}}+\mathrm{p}_{\mathrm{y}}=2(\mathrm{n}+1)$

$\therefore \quad p_{x}+p_{y}-2=2 n$

$(W)+(12): \quad p_{0}+3 \geqslant p_{x}+p_{y}$

$\because \quad$ (the smallest prime in $\mathrm{k}$ is $\mathbf{3}$ )

$\therefore \quad p_{y} \geqslant 3$

$\therefore(13) \rightarrow p_{0} \geqslant p_{x}$

$\because\left\{\Theta_{4}:\left\{\phi_{4}+\left\{3,5,7,11, \ldots, p_{2}, p_{1}\right\} \neq \triangleright 2(n+1)=p_{i}+p_{i i}\right\}\right.$ 
$\therefore\left\{(1)+(15) \rightarrow p_{x} \notin\left\{3,5,7,11, \ldots, p_{2}, p_{1}\right\} \rightarrow p_{x}>p_{1}\right\}$

【reason:

Hypothesis: $p_{x} \in\left\{3,5,7,11, \ldots, p_{2}, p_{1}\right\}$

$\because p_{x} \geqslant p_{y} \rightarrow\left\{p_{x}, p_{y}\right\} \in\left\{3,5,7,11, \ldots, p_{2}, p_{1}\right\}$

$\left\{3,5,7,11, \ldots, p_{2}, p_{1}\right\} \rightarrow \quad p_{x}+p_{y}=2(n+1) \quad$ Contradiction with (15)

$\therefore p_{x} \notin\left\{3,5,7,11, \ldots, p_{2}, p_{1}\right\} \rightarrow p_{x}>p_{1} 】$

$\{(14)+(16)\} \rightarrow p_{0} \geqslant p_{x}>p_{1}$

Because $p_{0}>P_{1}$, and the prime number: $p_{0}$ and $P_{1}$ adjacent.

$\left\{\mathrm{p}_{0}\right.$ and $\mathrm{P}_{1}$ adjacent. $+($ (17) $\} \rightarrow \therefore \mathrm{p}_{\mathrm{x}}=\mathrm{p}_{0}$

It is proved that: $\left(p_{x}=p_{0}\right)$, get: new ( $\alpha$ line).

new ( $\alpha$ line): $3 \rightarrow 5 \rightarrow 7 \rightarrow 7 \rightarrow 11 \rightarrow 13 \rightarrow 13 \rightarrow 17 \rightarrow 19 \rightarrow 19 \rightarrow \ldots . . p_{1} \rightarrow p_{0}$

$\therefore \quad \alpha$ line: Any two adjacent prime numbers satisfy: (lower) $\leqslant$ (upper).

New ( $\boldsymbol{\alpha}$ line) and ( $\boldsymbol{\alpha}$ line) are not contradictory. (lower) $\leqslant$ (upper).

At this time, it is completely proved that: $\Theta_{2} \quad$ (QED).

$\Theta_{1}$ and $\Theta_{2}$ are equivalent: $\Theta_{2}$ (QED) $\rightarrow \Theta_{1}$ (QED).

\section{Conclusion}

$\Theta_{1}$ and $\Theta_{2}$ are equivalent: $\Theta_{2}$ (QED) $\rightarrow \Theta_{1}$ (QED).

The new $\alpha$-line logic extends the old one. $\Theta_{1}, \Theta_{2} \quad$ (QED).

Complete the mathematical complete induction:

$\left\{\mathbf{A}_{\mathbf{1}}(\mathrm{K}\right.$ line:3 $)+($ a line $)($ lower $) \leqslant$ (upper) $\} \boldsymbol{\Theta}_{1}$ (QED)

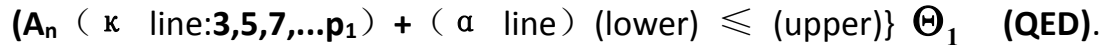

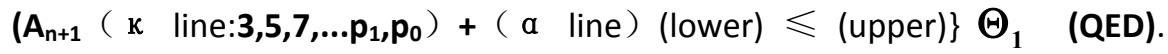

5

Statement:

1. There is no relevant data in my manuscript.

2. I cited references in my manuscript.

3. There is no conflict of interest. 
1 Bertrand Chebyshev theorem: if the integer $\mathbf{n}>\mathbf{3}$, then there is at least one prime $\mathbf{P}$, which conforms to $\mathbf{n}<\mathbf{p}<\mathbf{2 n - 2}$. Another slightly weaker argument is: for all integers $n$ greater than $\mathbf{1}$, there is at least one prime $\mathrm{P}$, which conforms to $\mathbf{n}<\mathbf{p}<\mathbf{2 n}$.

https://www. researchgate. net/publication/228592091 A Generalization o $\underline{f}$ Erdos's Proof of Bertrand-Chebyshev Theorem

2 The theorem of infinite number of primes: the $\mathrm{n}$ bit after each prime can always find another prime.

https://www. researchgate. net/publication/266044680 THE NUMBER OF PRIM ES IS INFINITE 